\title{
No-Go Theorem for Horizon-Shielded Self-Tuning Singularities
}

\author{
James M. Cline and Hassan Firouzjahi \\ Physics Department, McGill University, \\ 3600 University Street, Montréal, Québec, Canada H3A $2 T 8$
}

\begin{abstract}
We derive a simple no-go theorem relating to self-tuning solutions to the cosmological constant for observers on a brane, which rely on a singularity in an extra dimension. The theorem shows that it is impossible to shield the singularity from the brane by a horizon, unless the positive energy condition $(\rho+p \geq 0)$ is violated in the bulk or on the brane. The result holds regardless of the kinds of fields which are introduced in the bulk or on the brane, whether $Z_{2}$ symmetry is imposed at the brane, or whether higher derivative terms of the Gauss-Bonnet form are added to the gravitational part of the action. However, the no-go theorem can be evaded if the three-brane has spatial curvature. We discuss explicit realizations of such solutions which have both self-tuning and a horizon shielding the singularity.
\end{abstract}

PACS: $98.80 . \mathrm{Cq}$

McGill-00-18

\section{INTRODUCTION}

It is an interesting hypothesis that we might live on a 3-brane embedded in 5-dimensional Anti-deSitter space [1]; not only could this idea solve the hierarchy problem by explaining why mass scales on our brane are exponentially suppressed compared to $M_{\text {Planck }}$, but it might provide some phenomenological link between string theory, through the AdS/CFT correspondence, and TeV-scale physics [2].

At the same time, the braneworld scenario has created the hope of somehow circumventing Weinberg's nogo theorem for solving the cosmological constant problem using an adjustment mechanism, by virtue of introducing an extra dimension. Some attempts along these lines were made by [3:4], in which a scalar field in the bulk adjusted itself to yield a static solution to Einstein's equations, for a range of values of the brane tension. These solutions relied upon singular behavior of the scalar somewhere in the bulk, which was shown by ref. [5] to be simply a way of hiding the fine-tuning problem, since a proper treatment required insertion of a new brane at the singularity, whose tension must be tuned with respect to that of the visible brane. I In essence, the original selftuning idea was pretending to gain extra free parameters by no longer requiring boundary conditions to be satisfied at the boundary of the bulk where the singularity was appearing, and where a brane would normally have appeared.

A significant attempt to improve on this situation was made in ref. [7, 8]. Their idea was to render the singularity more physical by introducing a horizon between it and the visible brane, in the same way that the

\footnotetext{
${ }^{*}$ Moreover the original self-tuning solutions were shown to require a fine-tuning of the initial conditions in order to avoid motion of the singularity with respect to the brane [6].
}

Schwarzschild black hole singularity is hidden. In fact the bulk geometry is the AdS-Schwarzschild (or AdSReissner-Nordström in the case of a charged black hole) generalization of AdS, in which a singularity appears for all of 3-space at some position in the bulk. The singularity could thus be described as a black brane, though we will follow common usage and call it a black hole (BH).

The significance of the AdS-Schwarzschild solutions has become apparent in a number of works that deal with braneworld cosmology. In ref. [9] it was shown that this is the bulk solution which gives rise to the dark radiation term that was shown to be a possible addition to the Friedmann equation for the expansion of the brane. Ref. [10] subsequently identified the dark radiation as being identical to the thermal excitations of the CFT degrees of freedom in the context of the AdS/CFT correspondence. In ref. [11] it was shown that the bulk black hole must form in the early universe, since gravitational radiation emanating from the hot visible brane becomes infinitely dense as it falls toward the AdS horizon, for any cosmologically relevant initial brane temperature.

In contrast to these cosmological solutions, where the brane is moving away from the $\mathrm{BH}$ and thus seeing a bulk which becomes increasingly AdS-like (or alternatively, the dark radiation term in the brane Friedmann equation is redshifting away), ref. [7] finds a class of static solutions, so that the effect of the $\mathrm{BH}$ on the brane can be felt at arbitrarily late times. A very interesting application is that Lorentz invariance is broken, and gravitational signals travel with an average speed different from that of light on the brane. Most germane for the present work is that ref. [7] also finds self-tuning solutions with a horizon in the case of the charged (RN) black hole, where the mass and the charge of the $\mathrm{BH}$ adjusts itself to the energy density $\rho$ on the brane; but self-tuning and the horizon can coexist only if the positive energy condition is violated on the brane: $\rho<-p$.

Our motivation for the present work was to try to remove this seemingly unphysical restriction on the solu- 
tions and allow for positivity of the brane stress energy tensor. After various failed attempts we realized that the Einstein equations can be manipulated to show in a simple way why it is impossible to improve the situation by adding extra matter fields to the Lagrangian. This is our no-go theorem, which is given in the next section. In section III we show that the theorem also holds when the gravitational part of the action is supplemented with a particular higher-derivative correction, the GaussBonnet term. In section IV we discuss a way of evading the theorem: giving positive curvature to the $3-\mathrm{D}$ spatial hypersurfaces parallel to the brane. We generalize a solution of this type which was derived for the AdSSchwarzschild case to the case of nonvanishing charge and show the range of parameters where self-tuning and a horizon coexist. In section $\mathrm{V}$ we show that it is not possible to put the curvature into extra dimensions instead of the usual 3-D space, which would have been desirable for describing our flat universe. Conclusions are given in section VI.

\section{THE NO-GO THEOREM}

We begin with the following general action:

$$
S=\int d^{5} x \sqrt{-g}\left(\frac{R}{2 \kappa^{2}}+\mathcal{L}_{B}\right)+\int d^{4} x \sqrt{-g} \mathcal{L}_{b}
$$

where $\mathcal{L}_{B}$ and $\mathcal{L}_{b}$ are the Lagrangian densities in the bulk and on the brane respectively, and $\kappa^{2}=1 / M_{5}^{3}$ in terms of the 5-D Planck mass $M_{5}$. The ansatz for the metric, which includes the AdS, AdS-Schwarzschild or AdS-RN geometries, is

$$
d s^{2}=-h(r) d t^{2}+a(r) d \Sigma_{k}^{2}+h(r)^{-1} d r^{2}
$$

where $d \Sigma_{k}^{2}$ is the line element for a homogeneous 3-D space of constant spatial curvature with $k=0, \pm 1$. The 5 -D generalization of Birkhoff's theorem guarantees that this form for the bulk metric is a general solution (in the appropriate coordinate system) when there is only a cosmological constant [9, 12 or a $\mathrm{U}(1)$ gauge field [7] in the bulk. However we will also take it to be our ansatz for the metric when there are more general sources of stress-energy in the bulk. Since we are interested in static solutions, a coordinate system can always be found which puts the metric into the form (2). For definiteness we will write the 3 -D part of the metric as

$$
\begin{aligned}
d \Sigma_{k}^{2} & =\frac{d x^{2}+d y^{2}+d z^{2}}{\left(1+\frac{1}{4} k\left(x^{2}+y^{2}+z^{2}\right)\right)^{2}} \\
& \equiv \Sigma_{k}^{2}(x, y, z)\left(d x^{2}+d y^{2}+d z^{2}\right) .
\end{aligned}
$$

The nonzero components of the Einstein tensor are

$$
\begin{aligned}
G_{00} & =-\frac{3}{4} h\left(\frac{a^{\prime}}{a} h^{\prime}+2 h \frac{a^{\prime \prime}}{a}-4 \frac{k}{a h}\right) \\
G_{i i} & =a \Sigma_{k}^{2}\left(\frac{a^{\prime}}{a} h^{\prime}+h \frac{a^{\prime \prime}}{a}-\frac{h}{4}\left(\frac{a^{\prime}}{a}\right)^{2}+\frac{h^{\prime \prime}}{2}-\frac{k}{a h}\right) \\
G_{55} & =\frac{3}{4}\left(\left(\frac{a^{\prime}}{a}\right)^{2}+\frac{h^{\prime}}{h} \frac{a^{\prime}}{a}-4 \frac{k}{a h}\right)
\end{aligned}
$$

Next we will rewrite

$$
a(r)=a_{0} e^{-A(r)},
$$

where $a_{0}$ is an arbitrary constant with dimensions of (length $)^{2}$, and consider the following linear combination of the Einstein tensor components: $2 G_{00} / h+$ $2 G_{11} /\left(a \Sigma_{k}^{2}\right)$. Using the Einstein equations, $G_{m n}=$ $\kappa^{2}\left(T_{m n}^{B}+T_{\mu \nu}^{b} \sqrt{h} \delta_{m}^{\mu} \delta_{n}^{\nu} \delta\left(r-r_{0}\right)\right)$, where $\delta_{r}^{\mu} \equiv 0$, we obtain

$$
\begin{aligned}
& \left(h^{\prime}+h A^{\prime}\right)^{\prime}-\frac{3}{2} A^{\prime}\left(h^{\prime}+h A^{\prime}\right)+4 \frac{k}{a_{0}} e^{A} \\
& =2 \kappa^{2}\left(\frac{T_{00}^{B}}{h}+\frac{T_{11}^{B}}{a \Sigma_{k}^{2}}\right)+2 \kappa^{2}\left(\frac{T_{00}^{b}}{h}+\frac{T_{11}^{b}}{a \Sigma_{k}^{2}}\right) \sqrt{h} \delta\left(r-r_{0}\right)
\end{aligned}
$$

This can be integrated once since $\left(h^{\prime}+h A^{\prime}\right)^{\prime}-\frac{3}{2} A^{\prime}\left(h^{\prime}+\right.$ $\left.h A^{\prime}\right)$ is proportional to $\left(e^{-3 A / 2}\left(h^{\prime}+h A^{\prime}\right)\right)^{\prime}$. Using $Z_{2}$ symmetric boundary conditions at the brane to interpret the contribution of the delta function, we have

$$
\begin{aligned}
\left.\left(h^{\prime}+h A^{\prime}\right)\right|_{r} & =-2 \kappa^{2} e^{\frac{3}{2} A} \int_{r}^{r_{0}}\left(\frac{T_{00}^{B}}{h}+\frac{T_{11}^{B}}{a \Sigma_{k}^{2}}\right) e^{-\frac{3}{2} A} d r \\
& -\left.\kappa^{2} e^{\frac{3}{2}\left(A(r)-A\left(r_{0}\right)\right)}\left(\frac{T_{00}^{b}}{h}+\frac{T_{11}^{b}}{a \Sigma_{k}^{2}}\right) \sqrt{h}\right|_{r_{0}} \\
& +4 \frac{k}{a_{0}} e^{\frac{3}{2} A} \int_{r}^{r_{0}} e^{-\frac{1}{2} A} d r
\end{aligned}
$$

Let us for the moment consider the cases of vanishing or negative spatial curvature, $k=0$ or -1 . Then at the horizon $r=r_{H}, h=0$ and since the right hand side of eq. (7) is not positive - assuming that $-T_{0}^{0}+T_{1}^{1} \geq 0$, in accordance with positivity of the stress energy tensorwe conclude that $h^{\prime} \leq 0$ at the horizon. But the brane is located at a value $r_{0}>r_{h}$ beyond the horizon, by construction, and $h\left(r_{0}\right)$ must be positive for $t$ to be a timelike coordinate. This implies that $h^{\prime}\left(r_{h}\right)>0$ at the horizon, as shown in figure 1 , and in contradiction to eq. (7).

From the argument above, we can discern two ways of evading the no-go result. (1) Violate positivity of the stress-energy tensor in the bulk or on the brane, which would change the sign of either of the first two integrals in eq. (7). (2) Let the 3-D curvature be positive, making the third integral in (7) positive. In section IV we will consider the second possibility. For the moment, let us 
assume that neither (1) nor (2) is fulfilled, and moreover that the $3-\mathrm{D}$ curvature is zero, which is the most favorable case for horizon formation, apart from the positive curvature case.

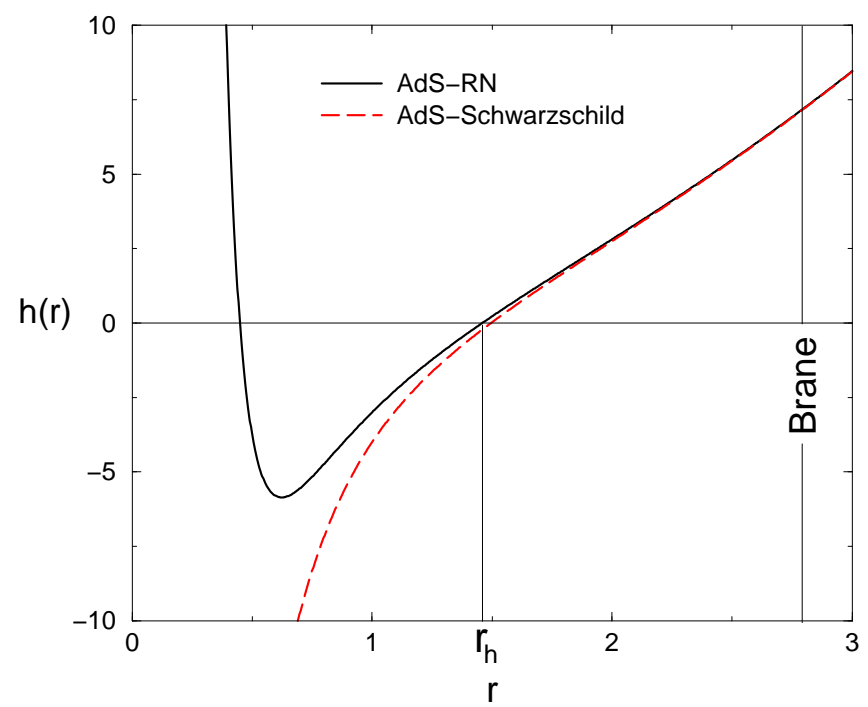

Figure 1: Required behavior for $h(r)$ near the horizon, $r=r_{h}$, for charged and for neutral black holes. Since the brane is at values of $r$ greater than $r_{h}$ and $h$ is supposed to be positive in this region, $h^{\prime}\left(r_{h}\right)$ must be positive.

To demonstrate the usefulness of our no-go theorem, we will now study two examples. Inclusion of a scalar field in the bulk along with gravity is the simplest one. In this case the stress-energy tensor contributions are

$$
\begin{aligned}
T_{m n}^{B} & =-g_{m n} V+\partial_{m} \Phi \partial_{n} \Phi-\frac{1}{2} g_{m n} \partial^{l} \Phi \partial_{l} \Phi \\
\left(T^{b}\right)_{n}^{m} & =\operatorname{diag}\left(-V_{0},-V_{0},-V_{0},-V_{0}, 0\right)
\end{aligned}
$$

where $V$ and $V_{0}$ are the potentials in the bulk and on the brane, respectively. Static solutions which respect Lorentz symmetry on the brane will be of the form $\Phi=$ $\Phi(r)$, i.e., dependent on the bulk coordinate only. Then from the above expressions we see that $\frac{T_{00}}{h}+\frac{T_{11}}{a}=0$ both in the bulk and on the brane and hence $\left(h^{\prime}+h A^{\prime}\right)=0$ everywhere in the bulk, as a consequence of eq. (7). In this case $h$ must be of the form $e^{-A}$, just like the metric component $a$. Thus the metric is explicitly Lorentz invariant, regardless of the choice of bulk or brane potentials, and there can be no horizon, with the possible exception of the usual AdS horizon at $r=\infty$, where $h$ and $a$ vanish together. In this case the situation is the same as was investigated in ref. 13], which found that self-tuning solutions where gravity is localized have a naked singularity.

As a second example, we consider the AdS-RN solution which ref. [7] investigated in depth. Here one introduces a $U(1)$ gauge field in addition to the negative vacuum energy in the bulk, giving the stress-energy tensor

$$
T_{m n}^{B}=-g_{m n} \Lambda-\frac{1}{4} g_{m n} F_{a b} F^{a b}+F_{m c} F_{n}^{c}
$$

The solution to the equations of motion is

$$
\begin{aligned}
h(r) & =\frac{r^{2}}{l^{2}}-\frac{\mu}{r^{2}}+\frac{Q^{2}}{r^{4}} \\
a(r) & =r^{2} \\
F_{t r} & =\frac{\sqrt{6} Q}{\kappa r^{3}}
\end{aligned}
$$

where $\mu$ and $Q$ are proportional to the black hole mass and charge, respectively, and $l^{-2}=-\frac{1}{6} \kappa^{2} \Lambda$. Subtituting this solution into the stress energy tensor we find

$$
\frac{T_{00}^{B}}{h}+\frac{T_{11}^{B}}{a}=\frac{6 Q^{2}}{\kappa^{2} r^{6}}>0
$$

From eq. (7) it is then clear that there is no possibility of having a horizon unless the positivity of the stress energy tensor on the brane is violated, i.e., $\rho+p<0$, where $\rho=-\left(T^{b}\right)_{0}{ }^{0}$ and $p=\left(T^{b}\right)_{1}{ }^{1}$. We note in passing that the jump conditions at the brane are

$$
\frac{\left[a^{\prime}\right]}{a}=-\frac{2}{3} \kappa^{2} \rho \sqrt{h^{-1}} ; \quad \frac{\left[h^{\prime}\right]}{h}=\frac{2}{3} \kappa^{2}(2 \rho+3 p) \sqrt{h^{-1}}
$$

where ['] denotes the discontinuity in the derivative across the brane. Since $h^{\prime}>0$ at the brane, its discontinuity assuming $Z_{2}$ symmetry is negative. Hence the brane tension must be positive; nevertheless $\rho+p$ is negative and the parameter $\omega \equiv p / \rho$ must be less than -1 .

It was recently proposed that adding a dilatonic coupling to the gauge field will improve the situation in such a way that the horizon could be outside of the brane [14, and the interior region containing the singularity is cut away when the $Z_{2}$ symmetry around the brane is imposed. In this situation, to satisfy the jump conditions (12), $\rho$ must be negative and $\omega \equiv p / \rho$ must be positive. This is in contrast to the AdS-RN situation where $\omega<-1$ was required, in contradiction to the positive energy condition. The authors of ref. 14 find that $\omega>0$ in their new solution, which may at first look like an improvement. However, when $\rho<0$, positivity of the stress-energy tensor actually requires that $\omega \leq-1$ (so that $\rho+p>0$ ), so we see that the problem still persists in their solution.

In fact we can easily extend our no-go theorem to the case where the brane is placed between the singularity and the horizon to show that no improvement is provided by this variation. Let us suppose there exists a bulk solution which is qualitatively like one of those shown in figure 2; these are the negatives of the normal AdS-Schwarzschild or AdS-RN solutions. In this case the brane should not be placed at $r>r_{h}$ because in this region $r$ is the timelike coordinate, and such a brane would

\footnotetext{
${ }^{\dagger}$ Ref. 114 does however remark upon the possibility of overcoming this problem when the curvature is $k=1$.
} 
not be static, as we would like for self-tuning, but instead would represent a time-dependent solution. Repeating the steps that led to eq. (『), we obtain

$$
\begin{aligned}
\left.\left(h^{\prime}+h A^{\prime}\right)\right|_{r} & =2 \kappa^{2} e^{\frac{3}{2} A} \int_{r_{0}}^{r}\left(\frac{T_{00}^{B}}{h}+\frac{T_{11}^{B}}{a \Sigma_{k}^{2}}\right) e^{-\frac{3}{2} A} d r \\
& +\left.\kappa^{2} e^{\frac{3}{2}\left(A(r)-A\left(r_{0}\right)\right)}\left(\frac{T_{00}^{b}}{h}+\frac{T_{11}^{b}}{a \Sigma_{k}^{2}}\right) \sqrt{h}\right|_{r_{0}} \\
& -4 \frac{k}{a_{0}} e^{\frac{3}{2} A} \int_{r_{0}}^{r} e^{-\frac{1}{2} A} d r
\end{aligned}
$$

The new condition (13) is identical to the old one (7) as far as the bulk contributions are concerned, but the sign of the brane contribution is changed because of the fact that the space is being cut away for $r<r_{0}$ rather than for $r>r_{0}$. Now when we apply (13) at the horizon, $r_{h}$, we find that $h^{\prime}\left(r_{h}\right)$ gets only positive contributions unless the $3-\mathrm{D}$ curvature is $k=1$, or positivity of $T_{\mu \nu}$ is violated. But figure 2 makes clear that $h^{\prime}\left(r_{h}\right)$ should be negative in this case, thus giving a contradition.

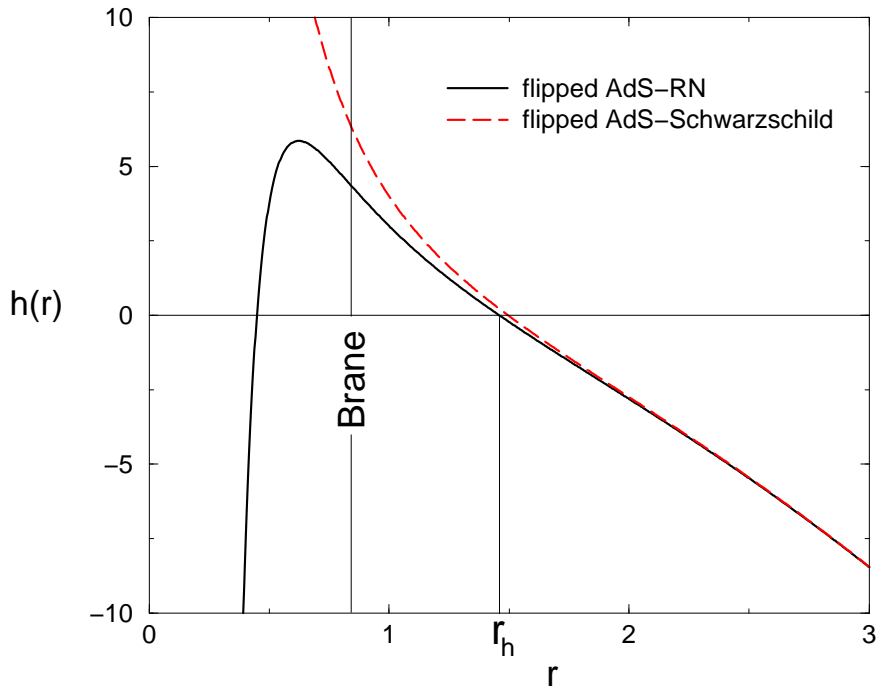

Figure 2. Qualitative behavior of hypothetical solutions which would require the brane to be placed between the horizon and the singularity.

\section{RELAXATION OF $Z_{2}$ SYMMETRY, AND HIGHER DERIVATIVE CORRECTIONS}

We have seen that nonvanishing $3-\mathrm{D}$ curvature $k$ can provide a way out of our no-go result, but one may wonder whether there are other loopholes. In this section we continue to leave $k=0$ and explore two possibilities which, as it turns out, do not provide any additional loophole. The first is to relax the $Z_{2}$ symmetry imposed at the brane, at $r=r_{0}$. We consider possible solutions in which

$$
h(r)= \begin{cases}h_{1}(r), & r<r_{0} \\ h_{2}(r), & r>r_{0}\end{cases}
$$

and similarly for $a_{i}(r)=a_{0} e^{-A_{i}(r)}$. The solution would have singularities on both sides of the brane, at positions $r=0$ and $r=r_{s}>r_{0}$, say. The hope would be to obtain horizons on both sides before the singularities are reached, at $r=r_{h 1}, r_{h 2}$, as illustrated in figure 3 .

Integrating eq. (6) in the bulk we get

$$
\begin{aligned}
\left.\left(h_{i}^{\prime}+h_{i} A_{i}^{\prime}\right)\right|_{r} & =2 \kappa^{2} e^{\frac{3}{2} A_{i}} \int_{r_{0}}^{r}\left(\frac{T_{00}^{B}}{h_{i}}+\frac{T_{11}^{B}}{a_{i}}\right) e^{-\frac{3}{2} A_{i}} d r \\
& +c_{i} e^{\frac{3}{2} A_{i}}
\end{aligned}
$$

where $c_{i}$ are the constants of integration determined by the jump conditions (12). Integrating eq. (6) across the brane gives

$$
\left(c_{2}-c_{1}\right) e^{\frac{3}{2} A\left(r_{0}\right)}=\left.\kappa^{2}\left(\frac{T_{00}^{b}}{h}+\frac{T_{11}^{b}}{a}\right) \sqrt{h}\right|_{r_{0}}
$$

which implies $\left(c_{2}-c_{1}\right)>0$. But at the first horizon, $r_{h_{1}}$, we need $h^{\prime}>0$ and eq. (15) thus requires $c_{1}>0$. Similarly getting $h^{\prime}<0$ at $r_{h_{2}}$ requires $c_{2}<0$. These two conditions give rise to the contradictory relation $\left(c_{2}-c_{1}\right)<0$; hence nothing is gained by relaxing $Z_{2}$ symmetry.

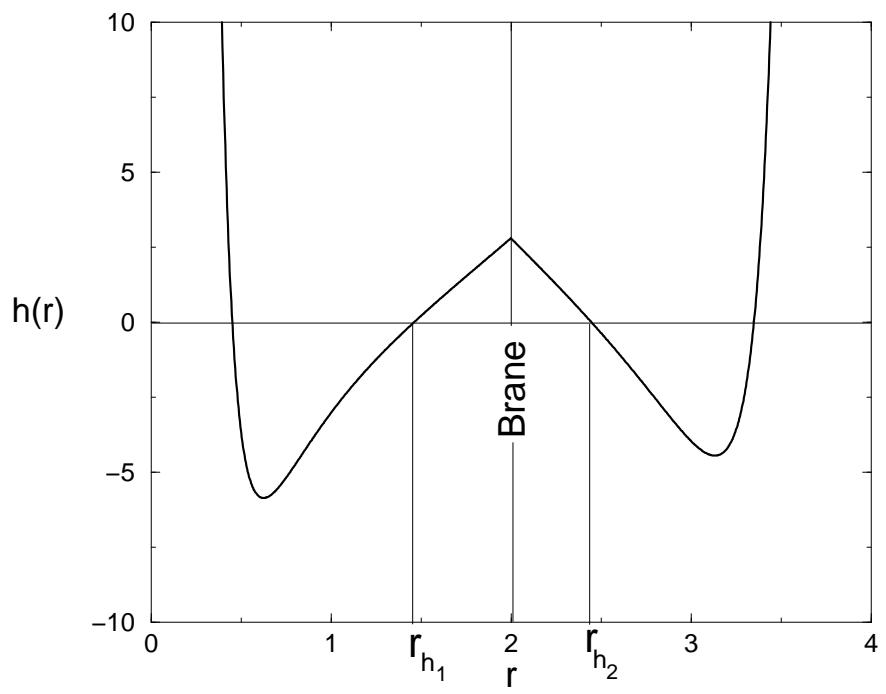

Figure 3. Qualitative behavior of possible solutions without $Z_{2}$ symmetry across the brane.

One might alternatively hope that adding higher derivative corrections to the action might circumvent the no-go theorem. The simplest such correction to the Einstein-Hilbert action is a Gauss-Bonnet term [15, 16], since this introduces extra powers of derivatives in the equations of motion without increasing the order of the equations. The action is

$$
S=\frac{1}{2 \kappa^{2}} \int d^{5} x \sqrt{-g}\left(R+\lambda\left(R^{2}-4 R_{a b} R^{a b}+R_{a b c d} R^{a b c d}\right)\right)
$$


where $\lambda$ is the coefficient of the new Gauss-Bonnet term. The modified Einstein equation is $\bar{G}_{m n}=\kappa^{2} T_{m n}$ where $\bar{G}_{m n}=G_{m n}+G_{m n}^{(\lambda)}$ and

$$
\begin{aligned}
G_{m n}^{(\lambda)} & =-\frac{\lambda}{2} g_{m n}\left(R^{2}-4 R_{a b} R^{a b}+R_{a b c d} R^{a b c d}\right) \\
& +2 \lambda\left(R R_{m n}-2 R_{m c} R_{n}^{c}+R_{m c d e} R_{n}^{c d e}+2 R^{c d} R_{m c d n}\right)
\end{aligned}
$$

The (00) and (ii) components of the new contribution to Einstein's tensor are

$$
\begin{aligned}
& G_{00}^{(\lambda)}=+\frac{3}{4} h \lambda\left(2\left(\frac{a^{\prime}}{a}\right)^{2} \frac{a^{\prime \prime}}{a} h^{2}-\left(\frac{a^{\prime}}{a}\right)^{4} h^{2}+\left(\frac{a^{\prime}}{a}\right)^{3} h^{\prime} h\right) \\
& G_{i i}^{(\lambda)}=-\frac{\lambda}{2}\left(2 \frac{a^{\prime \prime}}{a} \frac{a^{\prime}}{a}-\left(\frac{a^{\prime}}{a}\right)^{3}+\left(\frac{a^{\prime}}{a}\right)^{2} h^{\prime 2}+\left(\frac{a^{\prime}}{a}\right)^{2} h h^{\prime \prime}\right)
\end{aligned}
$$

As before, adding the (00) and (55) components of Einstein's equations gives a differential equation similar to eq. (6), but $\left(h^{\prime}+h A^{\prime}\right)$ must be replaced with $\left(h^{\prime}+h A^{\prime}\right)\left(1-\lambda h A^{\prime 2}\right)$. Integrating this gives

$$
\begin{aligned}
& \left.\left(h^{\prime}+h A^{\prime}\right)\left(1-\lambda h A^{\prime 2}\right)\right|_{r}= \\
& -2 \kappa^{2} e^{\frac{3}{2} A} \int_{r}^{r_{0}}\left(\frac{T_{00}^{B}}{h}+\frac{T_{11}^{B}}{a}\right) e^{-\frac{3}{2} A} d r^{\prime} \\
& -\left.\kappa^{2} e^{\frac{3}{2}\left(A(r)-A\left(r_{0}\right)\right)}\left(\frac{T_{00}^{b}}{h}+\frac{T_{11}^{b}}{a}\right) \sqrt{h}\right|_{r_{0}} .
\end{aligned}
$$

The new factor does not change anything with respect to achieving a horizon since $h$ vanishes there. Just as before, the theorem shows that at the putative horizon $h^{\prime}<0$, in contradiction to the required behavior.

\section{SOLUTIONS WITH 3-D CURVATURE}

In contrast to the negative results described above, it is possible to have both self-tuning and a horizon, with no violations of stress-energy positivity, when the spatial curvature is nonvanishing and positive. In fact, such a solution has already been obtained in ref. [17] in the case of the chargeless black hole with $k=1$. The authors of [17] did not identify their solution as being self-tuning, but if one regards the position of the brane along with the black hole mass as the properties of the solution which adjust to compensate for the brane tension, then it should indeed be considered as self-tuning. It is straightforward to apply the jump conditions to show that a static solution with

$$
h(r)=k+\frac{r^{2}}{l^{2}}-\frac{\mu}{r^{2}} ; \quad a(r)=r^{2},
$$

exists if $k=1$, if the brane is placed at the position satisfying

$$
\begin{aligned}
\kappa^{4} \rho^{2} & =\frac{18}{r_{0}^{2}}+\frac{36}{l^{2}} \\
\rho & >0 ; \quad p=-\rho
\end{aligned}
$$

(again using $l^{-2} \equiv-\frac{1}{6} \kappa^{2} \Lambda$ ) and if the black hole mass parameter is

$$
\mu=\frac{1}{2} r_{0}^{2} .
$$

Thus self-tuning works for the range of brane tensions $\rho>6 \kappa^{-2} l^{-1}$. The horizon is located at

$$
r_{h}^{2}=\frac{1}{2}\left(-l^{2}+\sqrt{l^{4}+2 r_{0}^{2} l^{2}}\right)
$$

which can be shown to be always between the singularity and the brane.

We can easily generalize the above solution to the case of a charged black hole, where

$$
h(r)=k+\frac{r^{2}}{l^{2}}-\frac{\mu}{r^{2}}+\frac{Q^{2}}{r^{4}}
$$

The jump conditions determine the mass and charge of the black hole to bef

$$
\begin{aligned}
\mu & =r_{0}^{4}\left(\frac{3}{l^{2}}+\frac{2}{r_{0}^{2}}-\frac{\kappa^{4} \rho^{2}}{12}\right) \\
Q^{2} & =r_{0}^{6}\left(\frac{2}{l^{2}}+\frac{1}{r_{0}^{2}}-\frac{\kappa^{4} \rho^{2}}{18}\right)
\end{aligned}
$$

The additional constant of integration, $Q$, introduces some freedom in the position of the brane, which now can have a range of values for a given brane tension. The condition for existence of the horizon becomes complicated because $h\left(r_{h}\right)=0$ is a cubic equation. It has real roots (hence a horizon) only if the following inequality coming from the discriminant of the cubic equation is satisfied:

$$
\left(\frac{2}{27} l^{4}+\frac{1}{3} \mu l^{2}+Q^{2}\right)^{2}<\frac{4}{27} l^{2}\left(\frac{1}{3} l^{2}+\mu\right)^{3}
$$

If we define

$$
\epsilon \equiv l^{2} / r_{0}^{2} ; \quad \eta \equiv \frac{1}{36} \kappa^{4} \rho^{2} l^{2}
$$

and use the expressions (26), this can be rewritten as $\left[1-\eta+\epsilon\left(1-\frac{1}{2} \eta\right)+\frac{1}{3} \epsilon^{2}+\frac{1}{27} \epsilon^{3}\right]^{2}<\left[1-\eta+\frac{2}{3} \epsilon+\frac{1}{9} \epsilon^{2}\right]^{3}$. Although this is hard to solve analytically, the results are shown numerically in figure 4 . The darkened region is where horizons exist for positive values of $Q^{2}$. The

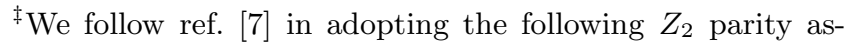
signments for the gauge field: $A_{r} \rightarrow+A_{r}, A_{t} \rightarrow-A_{t}$, so that no new jump condition arises for it.
} 
region above the wedge $(\eta>1+\epsilon / 2)$ corresponds to $Q^{2}<0$. The lower region has $Q^{2}$ exceeding the critical value beyond which the horizons are lost, resulting in a naked singularity. The boundary of this region can be approximated by the line $\eta \cong 1+\frac{12}{27} \epsilon$ (the approximation becoming exact as $\epsilon \rightarrow \infty$ ). Thus the allowed region for self-tuning with a horizon is given approximately by

$$
1+\frac{12}{27} \epsilon \lesssim \eta \leq 1+\frac{1}{2} \epsilon
$$

These solutions correspond to a brane with positive tension since the discontinuity of $h^{\prime}$ at $r_{0}$ is negative.

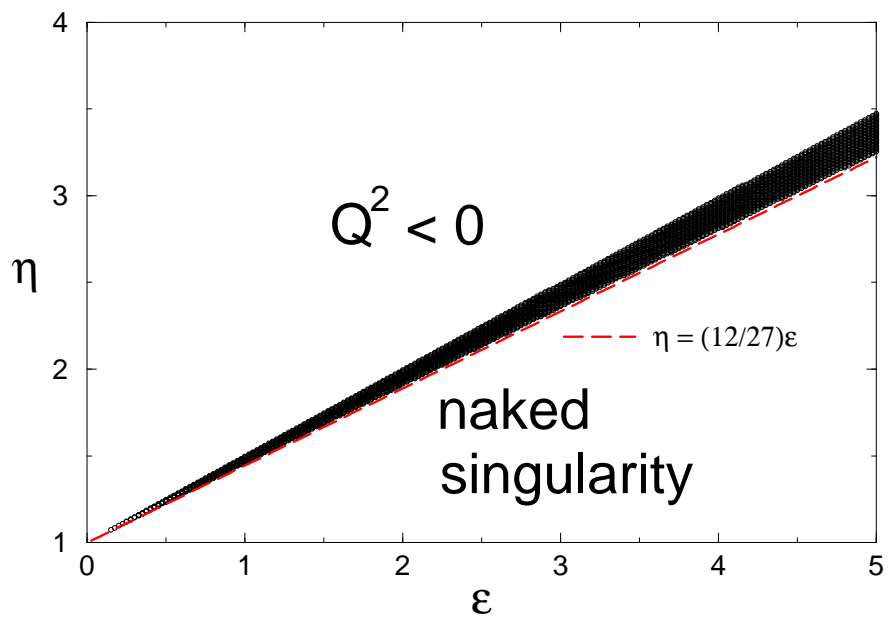

Figure 4: The dark wedge is the range of parameters (see eq. (28)) for which self-tuning with a horizon and with $Q^{2}>0$ occurs for the $k=+1$ AdS-RN solution. The upper limit corresponds to $Q=0$, and the lower one to the critical charge for which the horizons disappear.

We thus see that generalizing to $Q^{2}>0$ does not significantly relax the relation between the brane's tension and its position relative to the original chargeless solution, unless $r_{0} \ll l$. However the small $r_{0}$ regime is not very physical, because at distances much shorter than $l$, the AdS curvature scale, one expects higher derivative corrections to the gravitational action to alter the solution, so that one should not trust it in detail for $r \ll l$. Moreover, our universe would have to have very large values of $r_{0}$ in order to be nearly spatially flat.

We have also searched for static solutions with negative tension branes and $k=1$ in the AdS-RN case, where the brane is between the singularity and the horizon. These could in principle exist because of the inner horizon and the positivity of $h(r)$ in this region. However we do not find any such solutions. All those illustrated in figure 4 have positive tension branes located outside of the horizons. To arrive at this conclusion, we numerically evaluated the positions of the horizons, $r_{h_{i}}$, on a fine grid in the $\eta-\epsilon$ plane and checked whether $r_{0}$ was less than or greater than these values. The solutions of $h\left(r_{h}\right)=0$ are given by

$$
\frac{r_{h}^{2}}{l^{2}}=-\frac{1}{3}+2 \sqrt{\frac{A}{3}} \cos \theta
$$

where

$$
\begin{aligned}
A & =\frac{1}{3}+\frac{\mu}{l^{2}} ; \quad B=\frac{2}{27}+\frac{\mu}{3 l^{2}}+\frac{Q^{2}}{l^{4}} \\
\theta & =\frac{1}{3}\left(n \pi+\tan ^{-1} \sqrt{\frac{4 A^{3}}{27 B^{2}}-1}\right), \quad n=0,1, \ldots, 5
\end{aligned}
$$

(The three extraneous roots of the six given by this procedure were identified by substituting back into the original equation.) There are always two roots with positive $r_{h}^{2}$ and one unphysical one with negative $r_{h}^{2}$, except in the regions $\eta \geq 1+\epsilon / 2\left(Q^{2} \leq 0\right)$ (where there is only one horizon) and $\eta \lesssim 1+(12 / 27) \epsilon$ (where $Q^{2}$ exceeds the critical value for having any horizon). The physical values of $r_{h}$ are always less than that of the brane position, $r_{0}$.

\section{CURVED EXTRA DIMENSIONS}

Since we live in a universe that is nearly flat, it would be better if the effect of a large brane tension could be counteracted by the curvature of some small extra dimensions rather than that of the usual three. To explore whether this is possible, we consider the following ansatz for the metric, in which two extra dimensions with the geometry of a two-sphere of radius $\sqrt{b(r)}$ are introduced:

$$
\begin{aligned}
d s^{2}= & -h(r) d t^{2}+a(r) d \Sigma_{0}^{2}+h(r)^{-1} d r^{2} \\
& +b(r)\left(d \theta^{2}+\sin ^{2} \theta d \phi^{2}\right)
\end{aligned}
$$

The (00) and (ii) components of the Einstein tensor are

$$
\begin{aligned}
G_{00} & =-\frac{3}{4} h\left(\frac{a^{\prime}}{a} h^{\prime}+2 h \frac{a^{\prime \prime}}{a}\right) \\
& -\frac{h}{4}\left(6 \frac{a^{\prime}}{a} \frac{b^{\prime}}{b} h+2 \frac{b^{\prime}}{b} h^{\prime}-h\left(\frac{b^{\prime}}{b}\right)^{2}+4 \frac{b^{\prime \prime}}{b} h-\frac{4}{b}\right) \\
G_{i i} & =a\left(\frac{a^{\prime}}{a} h^{\prime}+h \frac{a^{\prime \prime}}{a}-\frac{h}{4}\left(\frac{a^{\prime}}{a}\right)^{2}+\frac{h^{\prime \prime}}{2}\right) \\
& +\frac{a}{4}\left(4 \frac{a^{\prime}}{a} \frac{b^{\prime}}{b} h+4 \frac{b^{\prime}}{b} h^{\prime}-h\left(\frac{b^{\prime}}{b}\right)^{2}+4 \frac{b^{\prime \prime}}{b} h-\frac{4}{b}\right)
\end{aligned}
$$

Although the last terms in $G_{00}$ and $G_{i i}$ show the effect of the positively curved extra dimensions, this effect cancels out of the relevant linear combination $\frac{G_{00}}{h}+\frac{G_{i i}}{a}$. Repeating the same steps that led to our previous no-go result gives

$$
\begin{gathered}
\left.\left(h^{\prime}+h A^{\prime}\right)\right|_{r}=-2 \frac{\kappa^{2}}{b} e^{\frac{3}{2} A} \int_{r}^{r_{0}} b\left(\frac{T_{00}^{B}}{h}+\frac{T_{11}^{B}}{a}\right) e^{-\frac{3}{2} A} d r \\
-\left.\kappa^{2} \frac{b\left(r_{0}\right)}{b(r)} e^{\frac{3}{2}\left(A(r)-A\left(r_{0}\right)\right)}\left(\frac{T_{00}^{b}}{h}+\frac{T_{11}^{b}}{a}\right) \sqrt{h}\right|_{r_{0}}
\end{gathered}
$$


Again we need $h^{\prime}\left(r_{h}\right)>0$, whereas the above expression shows that $h^{\prime}\left(r_{h}\right) \leq 0$, regardless of the curved extra dimensions. The latter thus do not provide any new loophole in our theorem.

\section{DISCUSSION}

By integrating a certain linear combination of the (00) and (ii) components of the Einstein equations, we have derived an enlightening constraint on the $g_{00}$ component of the metric when there is a black hole in a fivedimensional bulk, which is reminiscent of other consistency conditions that have been deduced for brane/bulk solutions 18. Our theorem explains why previous attempts to hide self-tuning singularities behind a horizon have had to resort to a brane equation of state which violates positivity of the stress-energy tensor. It shows that one could alternatively achieve the same effect by violating positivity in the bulk rather than on the brane. Indeed, in our search for self-tuning solutions with a horizon and with $p=-\rho$ on the brane, prior to deriving this theorem, we discovered that it is possible if the black hole charge has unphysical values with $Q^{2}<0$.

In our quest for loopholes to this constraint, we found that augmenting the gravitational action with higher powers of the curvature was unsuccessful, as was relaxing the $Z_{2}$ orbifold symmetry that is often assumed when cutting the bulk space off at the brane. Including positive spatial curvature for the 3 -D hypersurfaces provided a more successful way of evading the no-go theorem. We noted that the previously discovered solution of ref. 17] was an example of self-tuning with a horizon, and we generalized it by allowing the black hole to have a charge.

Unfortunately these positive curvature metrics do not provide a realistic solution to the cosmological constant problem because the curvature is related to the brane tension $\rho$ by $1 / r_{0}=C \sqrt{\rho} / M_{p}$, where $C$ is a number which lies within a narrow range of values of order 1 . In our universe, which is nearly flat, the same relation exists, where $\rho$ is the critical energy density. Therefore these "self-tuning" solutions can describe our universe only if the brane tension is on the order of the presently observed cosmological constant; this therefore constitutes fine-tuning after all. Precisely the same conclusion would hold if one tried to counteract the effect of a positive cosmological constant with positive curvature in a purely 4-D solution. This is nothing other than Einstein's static solution, which is known to be unstable against perturbations of the scale factor away from the special static value. It seems likely that the same problem will afflict the 5-D solutions as well. Moreover, we found that it was not possible to cancel the effect of the brane tension by shifting the positive curvature into small extra dimensions. Despite these somewhat negative conclusions however, given the notorious difficulty of the cosmological constant problem, the self-tuning approach with a horizon nevertheless seems deserving of further exploration.

Note added: the issue of shielding singularities in the bulk by horizons, and the difficulties of so doing, have been previously considered in ref. [19]. We thank S. Gubser for reminding us about this work.

We thank Christophe Grojean, Csaba Csaki, and Joshua Erlich for very helpful communications. H.F. thanks Neil Constable for his kind assistance with the Maple GRTensor package. J.C. acknowledges the generous hospitality of NORDITA during the completion of this work.

[1] L. Randall and R. Sundrum, Phys. Rev. Lett. 83, 3370 (1999) hep-ph/9905221; Phys. Rev. Lett. 83, 4690 (1999) hep-th/9906064.

[2] N. Arkani-Hamed, M. Porrati and L. Randall, "Holography and phenomenology," hep-th/0012148.

[3] N. Arkani-Hamed, S. Dimopoulos, N. Kaloper and R. Sundrum, Phys. Lett. B 480, 193 (2000) hhepth/0001197.

[4] S. Kachru, M. Schulz and E. Silverstein, Phys. Rev. D 62, 045021 (2000) hep-th/0001206.

[5] S. Forste, Z. Lalak, S. Lavignac and H. P. Nilles, JHEP 0009, 034 (2000) hep-th/0006139.

[6] P. Binetruy, J. M. Cline and C. Grojean, Phys. Lett. B 489, 403 (2000) hep-th/0007029.

[7] C. Csaki, J. Erlich and C. Grojean, Nucl. Phys. B 604, 312 (2001) hep-th/0012143.

[8] C. Csaki, J. Erlich and C. Grojean, "The cosmological constant problem in brane-worlds and gravitational Lorentz violations," gr-qc/0105114.

[9] P. Kraus, JHEP 9912, 011 (1999) hep-th/9910149.

[10] S. S. Gubser, Phys. Rev. D 63, 084017 (2001) hepth/9912001.

[11] A. Hebecker and J. March-Russell, hep-ph/0103214.

[12] P. Bowcock, C. Charmousis and R. Gregory, Class. Quant. Grav. 17, 4745 (2000) hep-th/0007177.

[13] C. Csaki, J. Erlich, C. Grojean and T. Hollowood, Nucl. Phys. B 584, 359 (2000) hep-th/0004133.

[14] C. Grojean, F. Quevedo, G. Tasinato and I. Zavala C., "Branes on charged dilatonic backgrounds: Self-tuning, Lorentz violations and cosmology," hep-th/0106120.

[15] H. Collins and B. Holdom, Phys. Rev. D 63, 084020 (2001) hep-th/0009127.

[16] O. Corradini and Z. Kakushadze, Phys. Lett. B 494, 302 (2000) hep-th/0009022.

[17] C. Gomez, B. Janssen and P. J. Silva, JHEP 0004, 027 (2000) hep-th/0003002.

[18] G. Gibbons, R. Kallosh and A. Linde, JHEP 0101, 022 (2001) hep-th/0011225;

F. Leblond, R. C. Myers and D. J. Winters, "Consistency conditions for brane worlds in arbitrary dimensions," hep-th/0106140.

[19] S. S. Gubser, "Curvature singularities: The good, the bad, and the naked," hep-th/0002160. 